\title{
Hidden symmetries of near-horizon extremal Kerr-AdS-NUT geometries
}

\author{
S. Sadeghian* \\ School of Physics, Institute for Research in Fundamental Sciences (IPM), \\ P.O. Box 19395-5531, Tehran, Iran
}

(Received 2 August 2018; published 17 October 2018)

\begin{abstract}
We study the hidden symmetries, the symmetries associated with Killing tensors, of the near-horizon geometry of odd-dimensional Kerr-AdS-NUT black holes in two limits: generic extremal and extremal vanishing horizon $(\mathrm{EVH})$ limits. Starting from a Kerr-AdS-NUT black hole in ellipsoidal coordinates which admit integrable geodesic equations, we obtain the near-horizon extremal and EVH geometries and their principal and Killing tensors by taking the near-horizon limit. We explicitly demonstrate that geodesic equations are separable and integrable on these near-horizon geometries. We also compute the constants of motion and read the Killing tensors of these near-horizon geometries from the constants of motion. As expected, they are the same as the Killing tensors given by taking the near-horizon limit.
\end{abstract}

DOI: 10.1103/PhysRevD.98.084031

\section{INTRODUCTION}

The exact symmetries in a general relativity framework are usually known as isometries that are given by Killing vectors. However, symmetries of a given metric can be generated by Killing tensors, as well. In this case, they are called hidden symmetries, as they are not manifested in the isometries. In some special cases, hidden symmetries reduce to the isometries when Killing tensors can be trivially written as the product of Killing vectors.

The symmetries of a metric are reflected in the motion of probe particles on that background metric, such that each of the Killing vectors or tensors gives a constant of motion. If the number of (independent) constants of motion is equal to the degrees of freedom of the probe particle, its equations are integrable. If it has more independent constants of motion, the system is superintegrable.

The Killing tensors of a four-dimensional Kerr black hole and its generalization to a $d$-dimensional Kerr-AdSNUT black hole have been studied in Refs. [1,2]. These symmetric second-rank tensors can be written as a contraction of two (antisymmetric) Killing-Yano tensors. In some sense, a Killing-Yano tensor is the "square root" of a Killing tensor. The Hodge dual of this Killing-Yano tensor is a closed conformal Killing two-form, called the principal tensor [3]. Using the eigenvectors of the nondegenerate principal tensor, one can find a coordinate basis in which

\footnotetext{
*ssadeghian@ipm.ir
}

Published by the American Physical Society under the terms of the Creative Commons Attribution 4.0 International license. Further distribution of this work must maintain attribution to the author(s) and the published article's title, journal citation, and DOI. Funded by SCOAP. geodesics, Klein-Gordon, Dirac, and Maxwell equations are separable in the probe limit [4-8]. (For a complete review, see Ref. [9].)

On the other hand, the near-horizon extremal geometry of a $d$-dimensional Kerr-AdS-NUT black hole is well understood. Similar to the other near-horizon extremal geometries of stationary black holes that show some universal properties including the attractor mechanism $[10,11]$ and symmetry enhancement $[12,13]$, its isometry group contains the $S O(2,1)$ group. Using this, one can describe the particle dynamics with conformal mechanics $[14,15]$. Moreover, there are special extremal black holes for which the symmetry enhances more in their nearhorizon geometries. They are called extremal vanishing horizon (EVH) black holes [16] and have been studied in Refs. $[17,18]$. In these cases, we find a common $S O(2,2)$ isometry group in the near-horizon EVH geometries [19].

In this paper, we try to answer this question: Are the hidden symmetries enhanced in the near-horizon limit? This has been questioned in Ref. [20] in four dimensions. Here, we start by studying the near-horizon geometry of odd-dimensional Kerr-AdS-NUT black holes in both extremal and EVH limits. ${ }^{1}$ Knowing the fact that the geodesic equations on black hole geometry are separable in ellipsoidal coordinates, we take the near-horizon limit in this coordinate system. The Killing tensors and their reduction to Killing vectors of near-horizon geometries has been studied in Refs. [21-23] only in the extremal case. Here, we extend it to the EVH case, as well. Also, we find the principal tensor of these near-horizon extremal/EVH geometries. Following the analysis in Refs. [24-26], we

\footnotetext{
${ }^{1}$ Such black holes can become EVH black holes only in odd dimensions [18].
} 
study the separability of timelike geodesic equations on near-horizon extremal/EVH geometries of Kerr-AdS-NUT black holes, explicitly. Finding the constants of motion, we infer that timelike geodesics on the corresponding background metrics are integrable.

\section{A BRIEF REVIEW OF THE KERR-ADS-NUT METRIC}

The metric of an odd-dimensional $(d=2 n+1)$ KerrAdS-NUT black hole in ellipsoidal coordinates [27] is

$$
\begin{aligned}
d s^{2}= & \sum_{\mu=1}^{n}\left(\frac{U_{\mu}}{X_{\mu}} d y_{\mu}^{2}\right. \\
& \left.+\frac{X_{\mu}}{U_{\mu}}\left[\frac{W}{\left(1-g^{2} y_{\mu}^{2}\right)} \frac{d t}{\Xi}-\sum_{i=1}^{n} \frac{a_{i}^{2} \gamma_{i}}{\left(a_{i}^{2}-y_{\mu}^{2}\right)} \frac{d \phi_{i}}{\varepsilon_{i}}\right]^{2}\right) \\
& -\frac{\prod_{k=1}^{n} a_{k}^{2}}{\prod_{\mu=1}^{n} y_{\mu}^{2}}\left(\frac{W}{\Xi} d t-\sum_{i=1}^{n} \gamma_{i} \frac{d \phi_{i}}{\varepsilon_{i}}\right)^{2},
\end{aligned}
$$

where the metric functions are ${ }^{2}$

$$
\begin{gathered}
U_{\mu}=\prod_{\nu=1}^{\prime}\left(y_{\nu}^{2}-y_{\mu}^{2}\right), \quad X_{\mu}=\frac{\left(1-g^{2} y_{\mu}^{2}\right)}{y_{\mu}^{2}} \prod_{k=1}^{n}\left(a_{k}^{2}-y_{\mu}^{2}\right)+2 M_{\mu}, \quad W=\prod_{\nu=1}^{n}\left(1-g^{2} y_{\nu}^{2}\right), \\
\gamma_{i}=\prod_{\nu=1}^{n}\left(a_{i}^{2}-y_{\nu}^{2}\right), \quad \varepsilon_{i}=a_{i} \Xi_{i} \prod_{k=1}^{n}\left(a_{i}^{2}-a_{k}^{2}\right), \quad \Xi_{i}=1-g^{2} a_{i}^{2}, \quad \Xi=\prod_{i=1}^{n} \Xi_{i} .
\end{gathered}
$$

Considering $y_{\mu}=\left(x_{\alpha}, i r\right)$ with $\alpha=1, \ldots, n-1$, one can see that $r$ is radial direction, and $x_{\alpha}$ and $\phi_{i}$ with $i=1, \ldots, n$ are related to the angular variables.

Note that $M_{n}$ is just equal to the mass parameter $M$, while the remaining $M_{\alpha}$ 's are NUT parameters, denoted by $L_{\alpha} . a_{i}$ denotes the rotation parameters.

Writing the metric in terms of $r$ coordinates, one can easily see that the horizon location is given by

$$
X_{n}\left(r=r_{h}\right)=\frac{\left(1+g^{2} r_{h}^{2}\right)}{r_{h}^{2}} \prod_{k=1}^{n}\left(a_{k}^{2}+r_{h}^{2}\right)-2 M=0 .
$$

The entropy and temperature of this horizon are

$$
\begin{aligned}
& S=\frac{A_{H}}{4 G_{N}}=\frac{m \mathcal{A}_{d-2}}{2 G_{N}\left(1+g^{2} r_{h}^{2}\right)}\left(\prod_{i=1}^{n} \frac{1}{\Xi_{i}}\right) r_{h}, \\
& T=\frac{\kappa_{H}}{2 \pi}=\frac{1}{2 \pi}\left[\sum_{i=1}^{n} \frac{r_{h}\left(1+g^{2} r_{h}^{2}\right)}{r_{h}^{2}+a_{i}^{2}}-\frac{1}{r_{h}}\right],
\end{aligned}
$$

in which $\mathcal{A}_{n}$ is the volume of a unit $n$-sphere, and $G_{N}$ is the $d$-dimension Newton's constant.

We note that $\frac{\partial}{\partial t}$ and $\frac{\partial}{\partial \phi_{i}}$ are the Killing directions, and the horizon is generated by the Killing vector

$$
\xi_{H}=\frac{\partial}{\partial t}-\sum_{i=1}^{n} \Omega^{i} \frac{\partial}{\partial \phi_{i}}
$$

where the horizon's angular velocity along each of the $\phi_{i}$ directions, $\Omega^{i}$, is given by

\footnotetext{
${ }^{2}$ The prime on the product symbol means that the factor which makes the product vanishing is removed.
}

$$
\Omega^{i}=\frac{a_{i}\left(1+g^{2} r_{h}^{2}\right)}{r_{h}^{2}+a_{i}^{2}}
$$

Additionally, this geometry has an $n$-number of secondrank Killing tensors. Killing tensors are symmetric, and a rank-r Killing tensor $K^{\mu_{1} \ldots \mu_{r}}$ satisfies

$$
\nabla^{(\nu} K^{\left.\mu_{1} \ldots \mu_{r}\right)}=0 .
$$

The Killing tensors of Kerr-AdS-NUT geometry, in the coordinate system in which that metric is written, are [2]

$$
\begin{aligned}
K_{(k)}= & \sum_{\mu=1}^{n}\left\{\frac{A_{\mu}^{(k)} X_{\mu}}{U_{\mu}}\left(\frac{\partial}{\partial y_{\mu}}\right)^{2}\right. \\
& \left.+\frac{A_{\mu}^{(k)} S_{\mu}}{y_{\mu}^{4} U_{\mu} X_{\mu}}\left[\frac{\partial}{\partial t}+\sum_{k=1}^{n} \frac{a_{k}\left(1-g^{2} y_{\mu}^{2}\right)}{\left(a_{k}^{2}-y_{\mu}^{2}\right)} \frac{\partial}{\partial \phi_{k}}\right]^{2}\right\} \\
& -\frac{c A^{(k)}}{\prod_{\nu=1}^{n} y_{\nu}^{2}}\left(\frac{\partial}{\partial t}+\sum_{k=1}^{n} \frac{1}{a_{k}} \frac{\partial}{\partial \phi_{k}}\right)^{2}, \quad k=0, \ldots, n-1,
\end{aligned}
$$

where $c=\prod_{i=1}^{n} a_{i}^{2}$ and

$$
\begin{aligned}
S_{\mu} & =\prod_{k=1}^{n}\left(a_{k}^{2}-y_{\mu}^{2}\right)^{2}, \quad A_{\mu}^{(k)}=\sum_{\substack{\nu_{1}<\nu_{2} \ldots<\nu_{k} \\
\nu_{1}, \ldots, \nu_{k} \neq \mu}}^{n} y_{\nu_{1}}^{2} y_{\nu_{2}}^{2} \ldots y_{\nu_{k}}^{2}, \\
A^{(k)} & =\sum_{\nu_{1}<\nu_{2} \ldots<\nu_{k}}^{n} y_{\nu_{1}}^{2} y_{\nu_{2}}^{2} \ldots y_{\nu_{k}}^{2} .
\end{aligned}
$$

One can simply check that $K_{(0)}$ is the inverse metric, as it trivially satisfies Eq. (7). After a coordinate transformation, the metric takes a simpler form (see Appendix A for the details): 


$$
\begin{aligned}
d s^{2}= & \sum_{\mu=1}^{n}\left[\frac{U_{\mu}}{X_{\mu}} d y_{\mu}^{2}+\frac{X_{\mu}}{U_{\mu}}\left(\sum_{j=0}^{n-1} A_{\mu}^{(j)} d \psi_{j}\right)^{2}\right] \\
& -\frac{c}{A^{(n)}}\left(\sum_{k=0}^{n} A^{(k)} d \psi_{k}\right)^{2} .
\end{aligned}
$$

The orthonormal vierbeins $\boldsymbol{e}^{\mu}, \hat{\boldsymbol{e}}^{\mu}(\mu=1, \ldots, n)$, and $\hat{\boldsymbol{e}}^{0}$ are

$\boldsymbol{e}^{\mu}=\left(\frac{U_{\mu}}{X_{\mu}}\right)^{\frac{1}{2}} d y_{\mu}, \quad \hat{\boldsymbol{e}}^{\mu}=\left(\frac{X_{\mu}}{U_{\mu}}\right)^{\frac{1}{2}} \sum_{j=0}^{n-1} A_{\mu}^{(j)} d \psi_{j}$,

$\hat{\boldsymbol{e}}^{0}=\left(\frac{c}{A^{(n)}}\right)^{\frac{1}{2}} \sum_{k=0}^{n} A^{(k)} d \psi_{k}$

and their dual vectors $\boldsymbol{e}_{\mu}, \hat{\boldsymbol{e}}_{\mu}, \hat{\boldsymbol{e}}_{0}$ are

$\boldsymbol{e}_{\mu}=\left(\frac{X_{\mu}}{U_{\mu}}\right)^{\frac{1}{2}} \partial_{y_{\mu}}, \quad \hat{\boldsymbol{e}}_{\mu}=\left(\frac{U_{\mu}}{X_{\mu}}\right)^{\frac{1}{2}} \sum_{k=0}^{n-1+\varepsilon} \frac{\left(-x_{\mu}^{2}\right)^{n-1-k}}{U_{\mu}} \partial_{\psi_{k}}$,

$\hat{\boldsymbol{e}}_{0}=\left(c A^{(n)}\right)^{-\frac{1}{2}} \partial_{\psi_{n}}$.

In these coordinates, the Killing tensors (8) are also simplified to

$$
K_{(k)}=\sum_{\mu=1}^{n} A_{\mu}^{(k)}\left[\boldsymbol{e}_{\mu} \boldsymbol{e}_{\mu}+\hat{\boldsymbol{e}}_{\mu} \hat{\boldsymbol{e}}_{\mu}\right]-A^{(k)} \hat{\boldsymbol{e}}_{0} \hat{\boldsymbol{e}}_{0}
$$

Moreover, this geometry has more rich structure that admits Killing-Yano tensors. We remind the reader that a Killing-Yano tensor of rank $q, Y_{\mu_{1} \ldots \mu_{q}}$, is an antisymmetric tensor and solves

$$
\nabla_{(\rho} Y_{\nu) \mu_{1} \ldots \mu_{q-1}}=0
$$

It is easy to show that the contraction of two Killing-Yano tensors in this way,

$$
K_{\mu \nu}=Y_{\mu \mu_{1} \ldots \mu_{s}} Y_{\nu}^{\mu_{1} \ldots \mu_{s}},
$$

gives a (symmetric) Killing tensor. The Hodge dual of a $(d-2)$-rank Killing-Yano tensor is a closed conformal Killing-Yano tensor of second rank, called a principal tensor, that satisfies

$$
h=\star Y, \quad \nabla_{\rho} h_{\mu \nu}=g_{\rho \mu} \xi_{\nu}-g_{\rho \nu} \xi_{\mu} .
$$

Here, $\xi$ is a primary Killing vector and is defined by

$$
\xi_{\mu}=\frac{-1}{(d-1)} \nabla^{\nu} h_{\mu \nu}
$$

Since the principal tensor, $h$, is closed, a potential $b$ is locally associated with it:

$$
h=d b .
$$

The importance of the principal tensor is that its orthogonal (nondegenerate) eigenvectors give a coordinate basis in which geodesic equations are separable.

The principal tensor of a Kerr-AdS-NUT black hole is

$h=\sum_{\mu=1}^{n} y_{\mu} d y_{\mu} \wedge\left(\sum_{k=0}^{n-1} A_{\mu}^{(k)} d \psi_{k}\right)=\sum_{\mu} y_{\mu} \boldsymbol{e}^{\mu} \wedge \hat{\boldsymbol{e}}^{\mu}$,

and its local potential $b$ is

$$
b=\frac{1}{2} \sum_{k=0}^{n-1} A^{(k+1)} d \psi_{k} .
$$

\section{NEAR-HORIZON EXTREMAL GEOMETRY}

The extremal limit of this black hole is given by vanishing the temperature in Eq. (4). In this case, the horizon becomes degenerate and

$$
\left.X_{n}^{\prime}\right|_{r=r_{h}}=0 .
$$

Note that $r_{h}$ is the solution to Eq. (3). The near-horizon transformations are also as follows:

$r=r_{h}+\lambda r_{h} \rho, \quad d t=\beta \frac{d \tau}{\lambda}, \quad d \phi_{i}=d \varphi_{i}+\Omega^{i} d t$,

$\beta=\frac{\prod_{i}\left(r_{h}^{2}+a_{i}^{2}\right)}{V r_{h}^{3}}$.

Applying these transformations and the constraint (21) to the metric (1) in the $\lambda \rightarrow 0$ limit, we find the near-horizon extremal geometry,

$$
\begin{aligned}
d s^{2}= & \frac{\tilde{U}_{n}}{V}\left(-\rho^{2} d \tau^{2}+\frac{d \rho^{2}}{\rho^{2}}\right) \\
& +\frac{c}{r_{h}^{2} \prod_{\alpha} x_{\alpha}^{2}}\left[\frac{2 \rho \tilde{U}_{n}}{r_{h} V} d \tau+\sum_{i=1}^{n} \tilde{\gamma}_{i} \frac{d \varphi_{i}}{\varepsilon_{i}}\right]^{2}+\sum_{\alpha=1}^{n-1} \frac{\tilde{U}_{\alpha}}{X_{\alpha}} d x_{\alpha}^{2} \\
& +\sum_{\alpha=1}^{n-1} \frac{X_{\alpha}}{\tilde{U}_{\alpha}}\left[\frac{2 r_{h} \rho \tilde{U}_{n}}{\left(r_{h}^{2}+x_{\alpha}^{2}\right) V} d \tau+\sum_{i=1}^{n} \frac{a_{i}^{2} \tilde{\gamma}_{i}}{\left(a_{i}^{2}-x_{\alpha}^{2}\right)} \frac{d \varphi_{i}}{\varepsilon_{i}}\right]^{2},
\end{aligned}
$$

where $V=-\left.\frac{1}{2} X_{n}^{\prime \prime}\right|_{r=r_{h}}$. The tilde over a function implies that it is evaluated at $r=r_{h}$.

\section{A. Principal and Killing tensors}

The Killing vectors of this geometry include the generators of rotation along $\varphi_{i}$,

$$
\zeta_{i}=\frac{\partial}{\partial \varphi_{i}}, \quad i=1 \ldots n
$$


and the generators of $\operatorname{sl}(2, R)$, as follows:

$$
\begin{aligned}
& \xi_{1}=\partial_{\tau} \\
& \xi_{2}=\tau \partial_{\tau}-\rho \partial_{\rho}, \\
& \xi_{3}=\left(\tau^{2}+\frac{1}{\rho^{2}}\right) \partial_{\tau}-2 \rho \tau \partial_{\rho}-\sum_{i=1}^{n} \frac{m_{i}}{\rho V} \frac{\partial}{\partial \varphi_{i}}
\end{aligned}
$$

where

$$
m_{i} \equiv \frac{4 a_{i} \Xi_{i} \prod_{j=1}^{n}\left(r_{h}^{2}+a_{j}^{2}\right)}{r_{h}\left(r_{h}^{2}+a_{i}^{2}\right)^{2}} .
$$

These $\xi_{i}$ 's satisfy $\operatorname{sl}(2, R)$ algebra:

$$
\left[\xi_{1}, \xi_{2}\right]=\xi_{1}, \quad\left[\xi_{1}, \xi_{3}\right]=2 \xi_{2}, \quad\left[\xi_{2}, \xi_{3}\right]=\xi_{3} .
$$

It is clear that $\zeta_{i}$ 's commute with $\xi_{i}$ 's. The Casimir of $\operatorname{sl}(2, R)$ algebra is

$$
\mathcal{I}=\frac{1}{2}\left(\xi_{1} \xi_{3}+\xi_{3} \xi_{1}\right)-\left(\xi_{2}\right)^{2}
$$

The nontrivial Killing tensors of this geometry have been studied earlier [22] and are given by

$$
\begin{aligned}
\tilde{K}_{(k)}= & -\frac{\tilde{A}_{n}^{(k)} V}{\tilde{U}_{n} \rho^{2}}\left(\frac{\partial}{\partial \tau}-\sum_{i=1}^{n} \frac{\rho m_{i}}{2 V} \frac{\partial}{\partial \varphi_{i}}\right)^{2}+\frac{\tilde{A}_{n}^{(k)} V \rho^{2}}{\tilde{U}_{n}}\left(\frac{\partial}{\partial \rho}\right)^{2} \\
& +\sum_{\alpha=1}^{n-1} \frac{\tilde{A}_{\alpha}^{(k)}\left(x_{\alpha}^{2}+r_{h}^{2}\right)^{2}}{x_{\alpha}^{4} X_{\alpha} \tilde{U}_{\alpha}}\left[\sum_{i=1}^{n} \frac{a_{i} \Xi_{i} \prod_{j=1}^{n}\left(x_{\alpha}^{2}-a_{j}^{2}\right)}{\left(x_{\alpha}^{2}-a_{i}^{2}\right)\left(r_{h}^{2}+a_{i}^{2}\right)} \frac{\partial}{\partial \varphi_{i}}\right]^{2} \\
& +\sum_{\alpha=1}^{n-1} \frac{\tilde{A}_{\alpha}^{(k)} X_{\alpha}}{\tilde{U}_{\alpha}}\left(\frac{\partial}{\partial x^{\alpha}}\right)^{2}-\frac{\tilde{A}^{(k)} r_{h}^{4}}{\tilde{A}^{(n)}}\left[\sum_{i=1}^{n} c_{i} \frac{\partial}{\partial \varphi_{i}}\right]^{2},
\end{aligned}
$$

where $k=0, \ldots, n-1$, and the functions $\tilde{A}_{\mu}^{(k)}, \tilde{A}^{(k)}$ are related to $A_{\mu}^{(k)}, A^{(k)}$ in Eq. (9) by setting $x_{n}=i r_{h}$. The constant $c_{i}$ 's are

$$
c_{i}=\frac{\Xi_{i} \prod_{j=1}^{n} a_{j}}{a_{i}\left(r_{h}^{2}+a_{i}^{2}\right)} .
$$

It is worth mentioning that these Killing tensors are invariant under the rotation and $s l(2, R)$ generated by Eqs. (24) and (25), respectively:

$$
\mathcal{L}_{\zeta_{i}} \tilde{K}_{(k)}=0, \quad \mathcal{L}_{\xi_{i}} \tilde{K}_{(k)}=0, \quad \forall i, k .
$$

The principal potential of four-dimensional near-horizon extremal Kerr-AdS-NUT geometry has been studied in Ref. [21]. However, for the $d$-dimensional case, it is not clear from Ref. [22] that this near-horizon geometry has the principal tensor or not. Apparently, the answer is negative, since the principal potential $b$ seems divergent in the near-horizon limit. Here, we will show that $b$ can still be well defined in the near-horizon limit if we use this freedom: $b$ is defined up to a shift like

$$
b \rightarrow b+C_{\mu} d x^{\mu}
$$

with constant $C_{\mu}$ 's, that does not affect the principal tensor $h$, as $h=d b$. We will show that the term which blows up in the near-horizon limit is a constant times $d t$ and can be absorbed using this freedom.

To apply the near-horizon transformations (22) to $b$ in Eq. (20), we should write it in terms of $d t$ and $d \phi_{i}$ using the coordinate transformation given in Appendix A. After a shift like $C d t$, it results in

$$
b=\left(C+b_{0}\right) d t+\sum_{i=1}^{n} b_{i} d \phi_{i},
$$

in which $b_{0}$ and $b_{i}$ are

$$
\begin{aligned}
& b_{0}=\frac{(-1)^{n+1}}{2 \Xi} \sum_{k=0}^{n-1} A^{(k+1)}\left(-g^{2}\right)^{k}, \\
& b_{i}=\frac{1}{2 \varepsilon_{i}} \sum_{k=0}^{n-1} A^{(k+1)}\left(-a_{i}^{2}\right)^{n-k},
\end{aligned}
$$

and $\varepsilon_{i}$ and $\Xi$ are defined in Eq. (2). In the near-horizon limit (22), $b$ takes the form

$$
\begin{aligned}
\tilde{b}= & {\left[C+\left.\left(b_{0}+\sum_{i=1}^{n} b_{i} \Omega^{i}\right)\right|_{r_{h}}\right.} \\
& \left.+\left.\left(b_{0}+\sum_{i=1}^{n} b_{i} \Omega^{i}\right)^{\prime}\right|_{r_{h}} \lambda r_{h} \rho+\mathcal{O}\left(\lambda^{2}\right)\right] \beta \frac{d \tau}{\lambda}+\sum_{i=1}^{n} \tilde{b}_{i} d \varphi_{i} .
\end{aligned}
$$

Here, the prime denotes a derivative with respect to the $r$ coordinate. As a result of the calculations in Appendix B II, $\left.\left(b_{0}+\sum_{i} b_{i} \Omega^{i}\right)\right|_{r_{h}}$ is constant and can be absorbed by the appropriate $C$. Therefore, it cancels the divergent term and results in

$$
\tilde{b}=\tilde{b}_{0} \rho d \tau+\sum_{i=1}^{n} \tilde{b}_{i} d \varphi_{i}
$$

where

$$
\tilde{b}_{0} \equiv \beta r_{h} \sum_{k=0}^{n-1}\left[\frac{\Omega^{i}}{\varepsilon_{i}}\left(-a_{i}^{2}\right)^{n-k}-\frac{(-1)^{n}}{\Xi}\left(-g^{2}\right)^{k}\right] \tilde{A}_{n}^{(k)} .
$$

We note that $\tilde{b}_{0}$ and $\tilde{b}_{i}$ are functions of $x_{\alpha}$ through $\tilde{A}_{n}^{(k)}$ and $\tilde{A}^{(k+1)}$, respectively, and do not depend on $\rho$. 
Such behavior has been investigated, with an explicit example in Appendix B II.

\section{B. Integrability of geodesic equations}

The simplest constant of motion for timelike geodesics is

$$
g^{a b} p_{a} p_{b}=-m_{0}^{2}
$$

Using the inverse metric of near-horizon geometry given by the $k=0$ of Eq. (29) and the projection of the Casimir element $\mathcal{I}$ onto the momentum space, we have

$$
\begin{gathered}
\frac{V}{\tilde{U}_{n}}\left(\mathcal{I}+\left[\sum_{i} \frac{m_{i} p_{i}}{2 V}\right]^{2}\right)+\frac{r_{h}^{4}}{\tilde{A}^{(n)}}\left[\sum_{i=1}^{n} c_{i} p_{i}\right]^{2} \\
-\sum_{\alpha=1}^{n-1}\left[\frac{\tilde{X}_{\alpha}}{\tilde{U}_{\alpha}} p_{\alpha}^{2}+\sum_{i, j=1}^{n} \frac{M_{\alpha}^{i j}}{\tilde{U}_{\alpha}} p_{i} p_{j}\right]=m_{0}^{2},
\end{gathered}
$$

where $M_{\alpha}^{i j}$ is defined by

$M_{\alpha}^{i j} \equiv \frac{\left(x_{\alpha}^{2}+r_{h}^{2}\right)^{2} \prod_{l=2}^{n}\left(a_{l}^{2}-x_{\alpha}^{2}\right)^{2} a_{i} \Xi_{i} a_{j} \Xi_{j}}{x_{\alpha}^{4} X_{\alpha}\left(a_{i}^{2}-x_{\alpha}^{2}\right)\left(a_{j}^{2}-x_{\alpha}^{2}\right)\left(r_{h}^{2}+a_{i}^{2}\right)\left(r_{h}^{2}+a_{j}^{2}\right)}$.

The angular Hamiltonian, $\mathcal{E}$, which is defined by

$$
\begin{aligned}
\mathcal{E} & \equiv V\left(\mathcal{I}+\frac{1}{4 V^{2}}\left[\sum_{i} m_{i} p_{i}\right]^{2}\right) \\
& =\frac{V}{\rho^{2}}\left(p_{0}-\sum_{i} \frac{\rho m_{i}}{2 V} p_{i}\right)^{2}-V \rho^{2} p_{\rho}^{2},
\end{aligned}
$$

can be rewritten in terms of angular variables using Eq. (39).

Similar to the analysis of Refs. $[24,26]$, we see that the Hamilton-Jacobi equations are also separable on the nearhorizon extremal geometry of an odd-dimensional KerrAdS-NUT black hole. Using the identity in Eq. (C3), the Eq. (39) can be conveniently rewritten as

$$
\sum_{\mu=1}^{n} \frac{1}{\prod_{\nu=1}^{n}{ }^{\prime}\left(x_{\nu}^{2}-x_{\mu}^{2}\right)}\left[R_{\mu}+W_{\mu}\right]=0,
$$

where

$$
\begin{aligned}
R_{n} & \equiv-\mathcal{E}, \\
R_{\alpha} & \equiv X_{\alpha} p_{\alpha}^{2}+\sum_{i, j=1}^{n} M_{\alpha}^{i j} p_{i} p_{j}, \\
W_{\mu} & \equiv m_{0}^{2}\left(-x_{\mu}^{2}\right)^{n-1}-\frac{r_{h}^{4}}{x_{\mu}^{2}}\left[\sum_{i=1}^{n} c_{i} p_{i}\right]^{2} .
\end{aligned}
$$

Recalling the identity $(\mathrm{C} 1)$, we can rewrite the expression (42) in the form

$$
\sum_{\mu=1}^{n} \frac{1}{\prod_{\nu=1}^{n}\left(x_{\nu}^{2}-x_{\mu}^{2}\right)}\left[R_{\mu}+W_{\mu}-\sum_{k=1}^{n-1} \nu_{k}\left(-x_{\mu}^{2}\right)^{n-1-k}\right]=0 .
$$

Here, $\nu_{k}$ 's are some arbitrary and independent constants which can be considered as constants of motion. To find the $\nu_{k}$ 's, we should reverse the equation:

$$
R_{\mu}+W_{\mu}=\sum_{k=1}^{n-1} \nu_{k}\left(-x_{\mu}^{2}\right)^{n-1-k} .
$$

By multiplying it with $\frac{\tilde{A}_{\mu}^{k}}{\prod_{\nu}{ }^{\prime}\left(x_{\nu}^{2}-x_{\mu}^{2}\right)}$, summing over $\mu$, and using the identities (C2) and (C4), we have

$$
\begin{aligned}
\nu_{k} & =-\frac{\tilde{A}_{n}^{(k)}}{\tilde{U}_{n}} \mathcal{E}+\sum_{\alpha=1}^{n-1} \frac{\tilde{A}_{\alpha}^{(k)} R_{\alpha}}{\tilde{U}_{\alpha}}-\frac{r_{h}^{4} A^{(k)}}{\tilde{A}^{(n)}}\left[\sum_{i=1}^{n} c_{i} p_{i}\right]^{2}, \\
k & =1, \ldots, n-1 .
\end{aligned}
$$

In addition to these $(n-1)$ constants of motion, $m_{0}^{2}$ is also a constant of motion. This can be considered as $\nu_{0}$ by the shift

$$
\nu_{k} \rightarrow \nu_{k}-m_{0}^{2} \delta_{k, 0} .
$$

We note to the reader that the range of $k$ was $[1, n-1]$ initially and did not include $k=0$. However, we extended it to include $k=0$.

Recalling Eq. (43) for the definition of $R_{\alpha}$ and $\mathcal{E}$ from (41), we have

$$
\begin{aligned}
\nu_{k}= & -\frac{V \tilde{A}_{n}^{(k)}}{\tilde{U}_{n} \rho^{2}}\left(p_{0}-\sum_{i} \frac{\rho m_{i}}{2 V} p_{i}\right)^{2}+\frac{V}{\tilde{U}_{n}} \rho^{2} p_{\rho}^{2} \tilde{A}_{n}^{(k)} \\
& +\sum_{\alpha=1}^{n-1} \frac{\tilde{A}_{\alpha}^{(k)}}{\tilde{U}_{\alpha}}\left[X_{\alpha} p_{\alpha}^{2}+\sum_{i, j=1}^{n} M_{\alpha}^{i j} p_{i} p_{j}\right] \\
& -\frac{r_{h}^{4} A^{(k)}}{\tilde{A}^{(n)}}\left[\sum_{i=1}^{n} c_{i} p_{i}\right]^{2},
\end{aligned}
$$

where $k$ runs over $[0, n-1]$ now. Considering these constants, $\nu_{k}$, as the contraction of Killing tensors, $K_{(k)}^{\mu \nu}$, with momentum $p_{\nu}$,

$$
\nu_{k}=K_{(k)}^{\mu \nu} p_{\mu} p_{\nu},
$$

and one can readily see that the resultant Killing tensors are the same as the Killing tensors in Eq. (29) that we obtained by taking the near-horizon limit. For instance, the Killing tensor related to $\nu_{0}$ is the metric itself (since $A^{(0)}=A_{\mu}^{(0)}=1$ ). 
In addition to these $n$ constants of motion made of Killing tensors, there are $n$ constants of motion associated with Killing vectors $\zeta_{i}$, of the form $\zeta_{i}^{\mu} p_{\mu}$, and two others from the Cartan and Casimir elements of $\operatorname{sl}(2, R)$. As a result of Eq. (31), these $2 n+2$ constants are Poisson commuting. However, all these constants of motion are not independent, and there is a constraint between them:

$$
\sum_{k=0}^{n-1} \nu_{k}\left(r_{h}^{2}\right)^{n-1-k}=-\mathcal{E}+r_{h}^{2}\left[\sum_{i=1}^{n} c_{i} p_{i}\right]^{2},
$$

as a combination of the corresponding Killing tensors can be written in terms of Killing vectors [21,22]. Altogether, geodesic equations on the near-horizon extremal geometry of a $(d=2 n+1)$-dimensional Kerr-AdS-NUT black hole have a $2 n+1$ independent, commuting constant of motion; therefore, they are integrable.

\section{NEAR-HORIZON EVH GEOMETRY}

In the previous section, it is assumed that the horizon area is nonzero. On the other hand, one can take a limit in which both horizon area and temperature vanish with the same rate. That is called the EVH limit. As one can readily see from the form of entropy in Eq. (4), the horizon area vanishes once $r_{h}$ goes to zero. The EVH limit of a KerrAdS-NUT black hole in odd dimensions has been studied in Ref. [18] with more details. It is given by a limit

$r_{h}=\rho_{0} \epsilon, \quad a_{1}=\tilde{a}_{1} \epsilon^{2}, \quad M=\frac{1}{2} \prod_{i=2}^{n} a_{i}^{2}+\tilde{M} \epsilon^{2}, \quad \epsilon \rightarrow 0$,

where the parameter $\tilde{m}$ is given by

$\tilde{M}=\frac{\rho_{0}^{2}}{2}\left(\frac{\tilde{a}_{1}^{2}}{\rho_{0}^{4}}-\lambda_{3}\right) \prod_{i=2}^{n} a_{i}^{2}, \quad \lambda_{3} \equiv-g^{2}-\sum_{i=2}^{n} \frac{1}{a_{i}^{2}}$.

(using the notations of Ref. [18] for $\lambda_{3}$ ).

To obtain the near-horizon limit of an EVH black hole, we apply the EVH limit (51) and the following transformations to the metric in Eq. (1):

$$
\begin{aligned}
t & =\beta_{3} \frac{\tau}{\gamma}, \quad r=r_{h}+\gamma \rho, \quad \phi_{1}=p \frac{\varphi}{\gamma}, \\
\phi_{i} & =\varphi_{i}+\Omega^{i} t, \quad 2 \leq i \leq n,
\end{aligned}
$$

where

$$
\begin{aligned}
& \beta_{3}=\frac{1}{V_{3}} \prod_{k=2}^{n} a_{k}^{2}, \quad p=\beta_{3} \sqrt{V_{3}}, \\
& V_{3}=-\left.\frac{1}{2} X_{n}^{\prime \prime}\right|_{r=r_{h}}=\left(-\lambda_{3}\right) \prod_{i=2}^{n} a_{i}^{2},
\end{aligned}
$$

and we assume that $\epsilon \ll \gamma$ in the $\epsilon, \gamma \rightarrow 0$ limit. In this case, the near horizon of an EVH black hole (NHEVH) reads as

$$
\begin{aligned}
d s_{N H}^{2}= & \frac{\tilde{U}}{V_{3}}\left(-\rho^{2} d \tau^{2}+\frac{d \rho^{2}}{\rho^{2}}+\rho^{2} d \varphi^{2}\right) \\
& +\sum_{\alpha=1}^{n-1}\left[\frac{\tilde{U}_{\alpha}}{\tilde{X}_{\alpha}} d x_{\alpha}^{2}+\frac{\tilde{X}_{\alpha}}{\tilde{U}_{\alpha}}\left(\sum_{i=2}^{n} \frac{a_{i}^{2} \tilde{\gamma}_{i}}{\left(a_{i}^{2}-x_{\alpha}^{2}\right) \tilde{\varepsilon}_{i}} d \varphi_{i}\right)^{2}\right],
\end{aligned}
$$

where $\tilde{\gamma}_{i}$ and $\tilde{\varepsilon}_{i}$ are

$$
\tilde{\gamma}_{i}=a_{i}^{2} \prod_{\alpha=1}^{n-1}\left(a_{i}^{2}-x_{\alpha}^{2}\right), \quad \tilde{\varepsilon}_{i}=a_{i} \prod_{k=2}^{n}{ }^{\prime}\left(a_{i}^{2}-a_{k}^{2}\right) .
$$

We note that the tilde on each quantity means that it is computed in the EVH and near-horizon limit. Therefore, the metric functions are

$$
\begin{gathered}
\tilde{U} \equiv \tilde{U}_{n}=\prod_{\alpha=1}^{n-1} x_{\alpha}^{2}, \quad \tilde{X}_{\alpha}=-\left(1-g^{2} x_{\alpha}^{2}\right) \prod_{k=2}^{n}\left(a_{k}^{2}-x_{\alpha}^{2}\right)+2 L_{\alpha} \\
\tilde{U}_{\alpha}=-x_{\alpha}^{2} \prod_{\beta=1}^{n-1^{\prime}}\left(x_{\beta}^{2}-x_{\alpha}^{2}\right), \quad S_{\alpha}=x_{\alpha}^{4} \prod_{k=2}^{n}\left(a_{k}^{2}-x_{\alpha}^{2}\right)^{2}
\end{gathered}
$$

One can check that this geometry is also a solution to the pure Einstein theory.

\section{A. Principal and Killing tensors}

The near-horizon geometry of Kerr-AdS-NUT black hole is given in the previous section. As is clear from Eq. (55), the metric includes an $\mathrm{AdS}_{3}$ factor and is invariant under the $S O(2,2)$ group. It can be viewed as two copies of $S L(2, R)$. In the coordinates

$$
v=\tau+\varphi, \quad u=\tau-\varphi,
$$

the generators of these two $\operatorname{SL}(2, R)$ are as follows:

$$
\begin{array}{lll}
H^{+}=\partial_{v}, & D^{+}=v \partial_{v}-\rho \partial_{\rho}, & K^{+}=v^{2} \partial_{v}+\frac{1}{\rho^{2}} \partial_{u}-2 \rho v \partial_{\rho}, \\
H^{-}=\partial_{u}, & D^{-}=u \partial_{u}-\rho \partial_{\rho}, & K^{-}=u^{2} \partial_{u}+\frac{1}{\rho^{2}} \partial_{v}-2 \rho u \partial_{\rho},
\end{array}
$$


and each of these sets satisfies $\operatorname{sl}(2, R)$ algebra:

$$
\begin{array}{ll}
{\left[H^{a}, D^{a}\right]=H^{a}, \quad\left[H^{a}, K^{a}\right]=2 D^{a},} \\
{\left[D^{a}, K^{a}\right]=K^{a}, \quad a=+,-.}
\end{array}
$$

The Casimir of each copy is

$$
\mathcal{I}^{ \pm}=\frac{1}{2}\left(H^{ \pm} K^{ \pm}+K^{ \pm} H^{ \pm}\right)-\left(D^{ \pm}\right)^{2}
$$

One can simply check that the Casimirs are equal.

Applying the EVH limit (51) and near-horizon limit (53) to the second-rank Killing tensors in Eq. (8) gives

$$
\begin{aligned}
K_{(k)}= & \tilde{A}^{(k)} \frac{V_{3}}{\tilde{U}}\left(-\frac{1}{\rho^{2}}\left(\partial_{\tau}\right)^{2}+\rho^{2}\left(\partial_{\rho}\right)^{2}+\frac{1}{\rho^{2}}\left(\partial_{\varphi}\right)^{2}\right), \\
& +\sum_{\alpha=1}^{n-1} \tilde{A}_{\alpha}^{(k)}\left(\frac{\tilde{X}_{\alpha}}{\tilde{U}_{\alpha}}\left(\frac{\partial}{\partial x^{\alpha}}\right)^{2}\right. \\
& \left.+\frac{S_{\alpha}}{\tilde{X}_{\alpha} \tilde{U}_{\alpha}}\left(\sum_{i=2}^{n} \frac{\Xi_{i}}{a_{i}\left(a_{i}^{2}-x_{\alpha}^{2}\right)} \frac{\partial}{\partial \varphi^{i}}\right)^{2}\right) .
\end{aligned}
$$

One can simply check that the $k=0$ case of them is just the inverse metric of the NHEVH of a Kerr-AdS-NUT black hole in Eq. (55).

The principal tensor $h$ can be read from its potential $b$, defined in Eq. (20), by applying the near-horizon and EVH limits from Eqs. (53) and (51), and taking the transformation (A5) into account. This gives

$$
\tilde{b}=\sum_{i=2}^{n} \tilde{b}_{i} d \varphi_{i}
$$

in which $\tilde{b}_{i}$ 's are the $b_{i}$ 's in Eq. (34) that should be computed in the near-horizon EVH limit.

\section{B. Integrability of geodesic equations}

Again, we start from the simplest constant of motion for geodesics, i.e.,

$$
g^{a b} p_{a} p_{b}=-m_{0}^{2}
$$

Using the inverse metric of near-horizon geometry given by the $k=0$ of Eq. (62) and the projection of the Casimir element $\mathcal{I}$ onto the momentum space, we have

$$
\begin{aligned}
& -\frac{V_{3}}{\tilde{U}} \mathcal{I}+\sum_{\alpha=1}^{n-1} \frac{\tilde{X}_{\alpha}}{\tilde{U}_{\alpha}} p_{\alpha}^{2}+\sum_{\alpha=1}^{n-1} \sum_{i, j=2}^{n} \frac{M_{\alpha}^{i j}}{\prod_{\beta=1}^{n-1 \prime}\left(x_{\beta}^{2}-x_{\alpha}^{2}\right)} p_{i} p_{j} \\
& \quad=-m_{0}^{2},
\end{aligned}
$$

where $M_{\alpha}^{i j}$ is defined by

$$
M_{\alpha}^{i j} \equiv \frac{\left(-x_{\alpha}^{2}\right) \prod_{l=2}^{n}\left(a_{l}^{2}-x_{\alpha}^{2}\right)^{2}}{X_{\alpha}\left(a_{i}^{2}-x_{\alpha}^{2}\right)\left(a_{j}^{2}-x_{\alpha}^{2}\right)} \frac{\Xi_{j}}{a_{i} a_{j}} .
$$

The angular Hamiltonian, $\mathcal{E}$, which is defined by

$$
\mathcal{E} \equiv V_{3} \mathcal{I}=V_{3}\left(\frac{1}{\rho^{2}}\left(p_{0}^{2}-p_{\varphi}^{2}\right)-\rho^{2} p_{\rho}^{2}\right),
$$

can be simplified using Eq. (65):

$$
\begin{aligned}
\mathcal{E}= & \left(\prod_{\alpha=1}^{n-1} x_{\alpha}^{2}\right)\left(\sum_{\alpha=1}^{n-1} \frac{\tilde{X}_{\alpha}}{\tilde{U}_{\alpha}} p_{\alpha}^{2}\right. \\
& \left.+\sum_{\alpha=1}^{n-1} \sum_{i, j=2}^{n} \frac{M_{\alpha}^{i j}}{\prod_{\beta=1}^{n-1 \prime}\left(x_{\beta}^{2}-x_{\alpha}^{2}\right)} p_{i} p_{j}+m_{0}^{2}\right) .
\end{aligned}
$$

Following the analysis of the separability of HamiltonJacobi equations in Refs. [24,26] reveals that the Hamilton-Jacobi equations are also separable on the near-horizon EVH geometry of a Kerr-AdS-NUT black hole in odd dimensions. Using the identity (C3), the angular Hamiltonian $\mathcal{E}$, given in Eq. (68), can be conveniently represented through

$$
\sum_{\alpha=1}^{n-1} \frac{1}{\prod_{\beta=1}^{n-1 \prime}\left(x_{\beta}^{2}-x_{\alpha}^{2}\right)}\left(R_{\alpha}(p, x)-\frac{\mathcal{E}}{x_{\alpha}^{2}}\right)=0
$$

where

$R_{\alpha}(p, x) \equiv-\frac{\tilde{X}_{\alpha}}{x_{\alpha}^{2}} p_{\alpha}^{2}+\sum_{i, j=2}^{n} M_{\alpha}^{i j} p_{i} p_{j}+m_{0}^{2}\left(-x_{\alpha}^{2}\right)^{n-2}$.

Recalling the identity $(\mathrm{C} 1)$, we can rewrite the expression (69) in a more useful form:

$$
\begin{aligned}
& \sum_{\alpha=1}^{n-1} \frac{1}{\prod_{\beta=1}^{n-1 \prime}\left(x_{\beta}^{2}-x_{\alpha}^{2}\right)}\left(R_{\alpha}(p, x)-\sum_{k=1}^{n-1} \nu_{k}\left(-x_{\alpha}^{2}\right)^{n-2-k}\right)=0, \\
& \nu_{n-1}=-\mathcal{E} .
\end{aligned}
$$

Here, $\nu_{k}$ 's are some arbitrary and independent constants which can be considered as constants of motion. To find $\nu_{k}$ 's, we should reverse the equation:

$$
R_{\alpha}(p, x)=\sum_{k=1}^{n-1} \nu_{k}\left(-x_{\alpha}^{2}\right)^{n-2-k} .
$$

By multiplication with $\frac{\tilde{A}_{\alpha}^{k}}{\prod_{\beta}^{\prime}\left(x_{\beta}^{2}-x_{\alpha}^{2}\right)}$, summation over $\alpha$, and using the identities (C2) and (C4), we have 
$\nu_{k}=-\frac{\tilde{A}^{(k)}}{\tilde{A}^{(n-1)}} \mathcal{E}+\sum_{\alpha=1}^{n-1} \frac{\tilde{A}_{\alpha}^{(k)} R_{\alpha}}{\prod_{\beta=1}^{n-1 \prime}\left(x_{\beta}^{2}-x_{\alpha}^{2}\right)}, \quad k=1, \ldots, n-2$.

This result can be rewritten by substituting $\mathcal{E}$ from Eq. (67) and noting that $\tilde{A}^{(n-1)}$ is just $\prod_{\alpha} x_{\alpha}^{2}$, as

$\nu_{k}=\frac{V_{3}}{\tilde{U}}\left(\rho^{2} p_{\rho}^{2}-\frac{1}{\rho^{2}}\left(p_{0}^{2}-p_{\varphi}^{2}\right)\right) \tilde{A}^{(k)}+\sum_{\alpha=1}^{n-1} \frac{\tilde{A}_{\alpha}^{(k)} R_{\alpha}}{\prod_{\beta=1}^{n-1 \prime}\left(x_{\beta}^{2}-x_{\alpha}^{2}\right)}$,

In addition to these $(n-1)$ constants of motion, $m_{0}^{2}$ is also a constant of motion. This can be considered as $\nu_{0}$ by the shift

$$
\nu_{k} \rightarrow \nu_{k}-m_{0}^{2} \delta_{k, 0}
$$

We note to the reader that the range of $k$ was $[1, n-1]$ initially and did not include $k=0$. However, we extended it to include $k=0$. Recalling Eq. (70) for the definition of $R_{\alpha}$, we have

$$
\begin{aligned}
\nu_{k}= & \frac{V_{3}}{\tilde{U}}\left(\rho^{2} p_{\rho}^{2}-\frac{1}{\rho^{2}}\left(p_{0}^{2}-p_{\varphi}^{2}\right)\right) \tilde{A}^{(k)} \\
& +\sum_{\alpha=1}^{n-1} \tilde{A}_{\alpha}^{(k)}\left(\frac{\tilde{X}_{\alpha}}{\tilde{U}_{\alpha}} p_{\alpha}^{2}+\frac{S_{\alpha}}{\tilde{X}_{\alpha} \tilde{U}_{\alpha}}\left(\sum_{i=2}^{n} \frac{\Xi_{i} p_{i}}{a_{i}\left(a_{i}^{2}-x_{\alpha}^{2}\right)}\right)^{2}\right),
\end{aligned}
$$

where $k$ now runs over $[0, n-1]$. Considering these constants, $\nu_{k}$, as the contraction of Killing tensors, $K_{(k)}^{\mu \nu}$, with momentum $p_{\mu}$,

$$
\nu_{k}=K_{(k)}^{\mu \nu} p_{\mu} p_{\nu},
$$

one can readily see that the resultant Killing tensors are the same as the Killing tensors in Eq. (62) that we obtained by taking the near-horizon limit.

Similar to the constraint (50) in the extremal case, we have

$$
\nu_{n-1}=-\mathcal{E},
$$

and all $\nu_{k}$ 's are not independent of the Casimir. Therefore, we have $(2 n+1)$ independent constants of motion in this case. So the geodesic equations on the near-horizon EVH Kerr-AdS-NUT geometry are also integrable and separable.

\section{DISCUSSION AND CONCLUSION}

In this work, we studied the principal and Killing tensors of near-horizon extremal and EVH geometries of a Kerr-AdS-NUT black hole in odd dimensions. The evendimensional case can be analyzed in a similar manner for the extremal case. Although the Killing tensors were given for the extremal case earlier [22,23], we improve the discussion of hidden symmetries by introducing the principal tensor for near-horizon extremal and EVH geometries. The principal tensor is a closed form and locally accompanied by a potential. Then, this potential is defined up to an exact form. In the near-horizon limit, we used this freedom to make the principal tensor finite. The existence of this tensor for a given metric makes geodesics, KleinGordon, Dirac, and Maxwell field equations separable on that background metric. We explicitly showed the separability of timelike geodesic equations on the mentioned near-horizon geometries.

Finding the constants of motion associated with the geodesics, one can read the Killing tensors of the background metric. We observed that the obtained Killing tensors in this way are the same as the Killing tensors given by taking the near-horizon limit.

One may also study the Penrose process and superradiance in these spacetimes and see if there are some distinct features due to taking special limits like in Ref. [28].

It is well known that the isometries enhance in the nearhorizon extremal limit, and Killing vectors have $\operatorname{sl}(2, R)$ algebra. However, there is no extra structure among the given second-rank Killing tensors and Killing vectors. Particularly, hidden symmetries (associated with the given second-rank Killing tensors) do not enhance in the near-horizon extremal or EVH limit. This statement should be revised for the equal angular momenta or for null geodesics.

In spite of the fact that the Casimir of $\operatorname{sl}(2, R)$ gives an extra constant of motion for the geodesic equations on nearhorizon extremal geometry, this problem is still integrable (not superintegrable), since there is a relation between the constants associated with the Killing tensors, Casimir and Killing vectors. One may expect that for the EVH case where we have $s o(2,2)$ as a subgroup of the isometries, we have more constants of motion. But this is not the case, because two constants that the Casimirs of $s o(2,2)$ provide are equal. Therefore, a geodesic problem on near-horizon EVH geometry of a Kerr-AdS-NUT black hole is also integrable.

These geometries have dual CFT descriptions from the AdS/CFT point of view. It would be interesting to find the meaning of the Killing tensors, hidden symmetries, and integrability of geodesics on the CFT side.

\section{ACKNOWLEDGMENTS}

The author is grateful to Hovhannes Demirchian, Armen Nersessian, and especially M. M. Sheikh-Jabbari for discussions during our previous collaborations. I also thank the conference on "Gravity-New perspectives from strings and higher dimensions," where the project was initiated. I learned hidden symmetries from David Kubiznak there and thank him. This work is partially supported by ICTP Program Network Scheme No. NT-04. 


\section{APPENDIX A: A USEFUL COORDINATE TRANSFORMATION}

Using the transformations

$$
\tilde{t}=\frac{t}{\Xi}, \quad \tilde{\phi}_{i}=\frac{\phi_{i}}{\varepsilon_{i}}
$$

on the Kerr-NUT-AdS metric (1) in odd dimensions $(D=2 n+1)$ and the definitions

$$
\begin{gathered}
a_{0}=\frac{1}{g}, \quad \Gamma_{I}=\prod_{\nu=1}^{n}\left(a_{I}^{2}-y_{\nu}^{2}\right), \quad 0 \leq I \leq n, \\
\tilde{\phi}_{0}=-g^{2 n} \tilde{t}, \quad X_{\mu}=\frac{g^{2}}{y_{\mu}^{2}} \prod_{I=0}^{n}\left(a_{I}^{2}-y_{\mu}^{2}\right)+2 M_{\mu},
\end{gathered}
$$

the metric can be written as

$$
\begin{aligned}
d s^{2}= & \sum_{\mu=1}^{n}\left\{\frac{U_{\mu}}{X_{\mu}} d y_{\mu}^{2}+\frac{X_{\mu}}{U_{\mu}}\left(\sum_{I=0}^{n} \frac{a_{I}^{2} \Gamma_{I} d \tilde{\phi}_{I}}{a_{I}^{2}-y_{\mu}^{2}}\right)^{2}\right\} \\
& -\frac{\left(\prod_{k=1}^{n} a_{k}^{2}\right)}{\left(\prod_{\mu=1}^{n} y_{\mu}^{2}\right)}\left(\sum_{I=0}^{n} \Gamma_{I} d \tilde{\phi}_{I}\right)^{2} .
\end{aligned}
$$

By using the relations

$$
\begin{gathered}
\Gamma_{I}=(-1)^{n} \sum_{k=0}^{n} A^{k}\left(-a_{I}^{2}\right)^{n-k}, \\
\left(a_{I}^{2}-y_{\mu}\right)^{-1} \Gamma_{I}=(-1)^{n-1} \sum_{k=0}^{n-1} A_{\mu}^{k}\left(-a_{I}^{2}\right)^{n-k-1},
\end{gathered}
$$

the metric (A3) takes the simpler form (10) if we define

$$
d \psi_{k}=\sum_{I=0}^{n}\left(-a_{I}^{2}\right)^{n-k} d \tilde{\phi}_{I}
$$

\section{APPENDIX B: PRINCIPAL TENSOR OF NEAR-HORIZON EXTREMAL GEOMETRY}

\section{Case study: 5D NHEMP}

In this part, we restrict our attention to the $g=0, L_{\alpha}=0$, and $d=5$ case-i.e., to the near-horizon extremal geometry of a five-dimensional Myers-Perry black hole [29]. This solution is described by two rotation parameters, $a_{1}$, $a_{2}$. After solving Eq. (3) for the horizon location in the extremal limit [Eq. (21)], we find that

$$
r_{h}^{2}=a_{1} a_{2}, \quad 2 M=\left(a_{1}+a_{2}\right)^{2} .
$$

The near-horizon metric is

$$
\begin{aligned}
d s^{2}= & -\frac{\tilde{U} \rho^{2} d \tau^{2}}{V}+\frac{\tilde{U} d \rho^{2}}{V \rho^{2}}+\frac{\tilde{U}_{1}(x)}{X_{1}(x)} d x^{2}+\frac{a_{1}^{2} a_{2}^{2}}{r_{h}^{2} x^{2}}\left(2 \frac{\tilde{U} \rho d \tau}{V r_{h}}+\frac{\tilde{\gamma}_{1}(x) d \varphi_{1}}{\varepsilon_{1}}+\frac{\tilde{\gamma}_{2}(x) d \varphi_{2}}{\varepsilon_{2}}\right)^{2} \\
& +\frac{X_{1}(x)}{\tilde{U}_{1}(x)}\left(\frac{2 r_{h} \tilde{U} \rho d \tau}{V\left(r_{h}^{2}+x^{2}\right)}+\frac{a_{1}^{2} \tilde{\gamma}_{1}(x) d \varphi_{1}}{\left(a_{1}^{2}-x^{2}\right) \varepsilon_{1}}+\frac{a_{2}^{2} \tilde{\gamma}_{2}(x) d \varphi_{2}}{\left(a_{2}^{2}-x^{2}\right) \varepsilon_{2}}\right)^{2}
\end{aligned}
$$

and its functions are

$$
\begin{aligned}
\tilde{U} & =\left(x^{2}+a_{1} a_{2}\right), \quad \Xi=1, \quad V=4, \\
\tilde{\gamma}_{1}(x) & =\left(a_{1}^{2}-x^{2}\right)\left(a_{1}+a_{2}\right) a_{1}, \quad \varepsilon_{1}=a_{1}\left(a_{1}^{2}-a_{2}^{2}\right), \\
\tilde{\gamma}_{2}(x) & =\left(a_{2}^{2}-x^{2}\right)\left(a_{1}+a_{2}\right) a_{2}, \quad \varepsilon_{2}=-a_{2}\left(a_{1}^{2}-a_{2}^{2}\right), \\
\tilde{U}_{1}(x) & =-\left(x^{2}+a_{1} a_{2}\right), \quad X_{1}(x)=x^{-2}\left(a_{1}^{2}-x^{2}\right)\left(a_{2}^{2}-x^{2}\right) .
\end{aligned}
$$

As discussed in Sec. III A, this geometry has two second-rank Killing tensors. One of them, $K_{(0)}$, is the metric itself, and another is

$$
\begin{aligned}
K_{(1)}= & \frac{-4 x^{2}}{\left(a_{1} a_{2}+x^{2}\right) \rho^{2}}\left(\partial_{\tau}\right)^{2}+\frac{4 \rho^{2} x^{2}}{a_{1} a_{2}+x^{2}}\left(\partial_{\rho}\right)^{2}+\frac{\left(a_{1}{ }^{2}-x^{2}\right)\left(a_{2}{ }^{2}-x^{2}\right) a_{1} a_{2}}{x^{2}\left(a_{1} a_{2}+x^{2}\right)}\left(\partial_{x}\right)^{2} \\
& -\frac{a_{2}\left(a_{1}{ }^{3} a_{2}{ }^{2}+a_{1}{ }^{3} x^{2}-2 a_{1}{ }^{2} a_{2}{ }^{3}+4 a_{1}{ }^{2} a_{2} x^{2}-a_{1} a_{2}{ }^{4}-a_{1} a_{2}{ }^{2} x^{2}-2 a_{2} x^{4}\right)}{\left(a_{1}{ }^{2}-x^{2}\right)\left(a_{1}+a_{2}\right)^{2}\left(a_{1} a_{2}+x^{2}\right)}\left(\partial_{\varphi_{1}}\right)^{2} \\
& +\frac{a_{1}\left(a_{1}{ }^{4} a_{2}+2 a_{1}{ }^{3} a_{2}{ }^{2}-a_{1}{ }^{2} a_{2}{ }^{3}+a_{1}{ }^{2} a_{2} x^{2}-4 a_{1} a_{2}{ }^{2} x^{2}+2 a_{1} x^{4}-a_{2}{ }^{3} x^{2}\right)}{\left(a_{2}{ }^{2}-x^{2}\right)\left(a_{1}+a_{2}\right)^{2}\left(a_{1} a_{2}+x^{2}\right)}\left(\partial_{\varphi_{2}}\right)^{2} \\
& +\frac{2 \sqrt{a_{2}} x^{2}}{\sqrt{a_{1}}\left(a_{1} a_{2}+x^{2}\right) \rho}\left(\partial_{\tau}\right)\left(\partial_{\varphi_{1}}\right)+\frac{2 \sqrt{a_{1}} x^{2}}{\sqrt{a_{2}}\left(a_{1} a_{2}+x^{2}\right) \rho}\left(\partial_{\tau}\right)\left(\partial_{\varphi_{2}}\right)+\frac{2 a_{1}{ }^{2} a_{2}{ }^{2}-a_{1}{ }^{2} x^{2}-a_{2}{ }^{2} x^{2}}{\left(a_{1}+a_{2}\right)^{2}\left(a_{1} a_{2}+x^{2}\right)}\left(\partial_{\varphi_{1}}\right)\left(\partial_{\varphi_{2}}\right) .
\end{aligned}
$$


The angular velocities are

$$
\Omega^{1}=\Omega^{2}=\frac{1}{\left(a_{1}+a_{2}\right)} .
$$

Then, $b_{0}$ and the $b_{i}$ 's in Eq. (34) are equal to

$$
\begin{gathered}
b_{0}=-\frac{1}{2 \Xi}\left(g^{2} r^{2} x^{2}+x^{2}-r^{2}\right), \\
b_{1}=\frac{1}{2 \varepsilon_{1}}\left[a_{1}^{2} r^{2} x^{2}+a_{1}^{4}\left(x^{2}-r^{2}\right)\right], \\
b_{2}=\frac{1}{2 \varepsilon_{2}}\left[a_{2}^{2} r^{2} x^{2}+a_{2}^{4}\left(x^{2}-r^{2}\right)\right] .
\end{gathered}
$$

By applying the near-horizon transformation,

$$
\begin{aligned}
& r=r_{h}+\lambda r_{h} \rho, \quad d t=\beta \frac{d \tau}{\lambda}, \quad d \phi_{i}=d \varphi_{i}+\Omega^{i} d t, \\
& \beta=\frac{\left(a_{1}+a_{2}\right)^{2}}{4 r_{h}},
\end{aligned}
$$

to the principal potential $b$ in Eqs. (33) and (34), it is easy to see that

$$
\left.\left(b_{0}+\Omega^{1} b_{1}+\Omega^{2} b_{2}\right)\right|_{r_{h}}=\frac{a_{1}^{2} a_{2}^{2}}{2\left(a_{1}+a_{2}\right)^{2}},
$$

which is constant. Therefore, choosing $C=-\frac{a_{1}^{2} a_{2}^{2}}{2\left(a_{1}+a_{2}\right)^{2}}$ will remove the divergent term of $b$ in the near-horizon limit. Then, we get

$$
\tilde{b}=\tilde{b}_{0} \rho d \tau+\tilde{b}_{1} d \varphi_{1}+\tilde{b}_{2} d \varphi_{2}
$$

where

$$
\begin{aligned}
& \left.\tilde{b}_{0} \equiv \beta r_{h} \frac{d}{d r}\left(b_{0}+\Omega^{1} b_{1}+\Omega^{2} b_{2}\right)\right|_{r_{h}}=\frac{\sqrt{a_{1} a_{2}}\left(x^{2}+a_{1} a_{2}\right)}{4}, \\
& \tilde{b}_{1}=-\frac{a_{1}{ }^{2}\left(a_{1}{ }^{2} a_{2}-a_{1} x^{2}-x^{2} a_{2}\right)}{2\left(a_{1}{ }^{2}-a_{2}{ }^{2}\right)}, \\
& \tilde{b}_{2}=\frac{a_{2}{ }^{2}\left(a_{1} a_{2}{ }^{2}-a_{1} x^{2}-x^{2} a_{2}\right)}{2\left(a_{1}{ }^{2}-a_{2}{ }^{2}\right)} .
\end{aligned}
$$

The principal tensor, $h=d b$, reads

$$
\begin{aligned}
h= & -\frac{\sqrt{a_{1} a_{2}}}{4}\left(a_{1} a_{2}+x^{2}\right) d \tau \wedge d \rho-\frac{\rho x \sqrt{a_{1} a_{2}}}{2} d \tau \wedge d x \\
& +\frac{x}{\left(a_{1}-a_{2}\right)} d x \wedge\left(a_{1}^{2} d \varphi_{1}-a_{2}^{2} d \varphi_{2}\right) .
\end{aligned}
$$

The Hodge dual of $h$ gives a Killing-Yano tensor of this form:

$$
\begin{aligned}
\star h= & \frac{\left(a_{1}+a_{2}\right) x a_{1} a_{2}}{a_{1}-a_{2}} d \tau \wedge d \rho \wedge d \varphi_{1}-\frac{\left(a_{1} a_{2}+x^{2}\right)\left(a_{1}^{2}-x^{2}\right) \sqrt{a_{1} a_{2}}}{4\left(a_{1}-a_{2}\right)} d \tau \wedge d \rho \wedge d \varphi_{2} \\
& +\frac{\left(a_{1}+a_{2}\right) \rho a_{1} \sqrt{a_{1} a_{2}} x}{2\left(a_{1}-a_{2}\right)} d \tau \wedge d x \wedge d \varphi_{1}-\frac{\left(a_{1}+a_{2}\right) \rho a_{2} \sqrt{a_{1} a_{2}} x}{2\left(a_{1}-a_{2}\right)} d \tau \wedge d x \wedge d \varphi_{2} \\
& +\frac{\left(a_{1} a_{2}+x^{2}\right)\left(a_{2}^{2}-x^{2}\right) \sqrt{a_{1} a_{2}}}{4\left(a_{1}-a_{2}\right)} d x \wedge d \varphi_{1} \wedge d \varphi_{2} .
\end{aligned}
$$

The Killing tensor made of this Killing-Yano tensor, using Eq. (15), is proportional to $K_{(1)}$ given by Eq. (B4).

\section{Generic odd dimensions}

As discussed in Sec. IVA, the principal potential, $b$, is divergent in the near-horizon limit. However, it is defined up to a shift of the form

$$
b \rightarrow b+C_{\mu} d x^{\mu}
$$

with constant $C_{\mu}$ 's. This shift does not affect $h=d b$. We use this freedom to make $b$ finite in the near-horizon limit. The divergent term arises from $d t \rightarrow d \tau / \lambda$. In the following, we show that its coefficient, which is equal to $\left.\left(b_{0}+\sum_{i} b_{i} \Omega^{i}\right)\right|_{r_{h}}$, is a constant. We start by substituting $b_{0}$ and the $b_{i}$ 's from Eq. (34):

$$
\begin{aligned}
B & \left.\equiv\left(b_{0}+\sum_{i=1}^{n} b_{i} \Omega^{i}\right)\right|_{r_{h}} \\
& =-\frac{\left(r_{h}^{2}+a_{0}^{2}\right)}{2} \sum_{J=0}^{n}\left(\frac{\sum_{k=0}^{n-1}\left(-a_{J}^{2}\right)^{n-k} A^{(k+1)}}{\left(r_{h}^{2}+a_{J}^{2}\right) \prod_{l=1}^{n}{ }^{\prime}\left(a_{i}^{2}-a_{l}^{2}\right)}\right) .
\end{aligned}
$$

The summation over $k$ can be easily done by changing $k=u-1$ :

$$
\begin{aligned}
S_{1} & \equiv \sum_{u=1}^{n}\left(-a_{J}^{2}\right)^{n+1-u} A^{(u)} \\
& =\left(-a_{J}^{2}\right)\left[\sum_{u=0}^{n}\left(-a_{J}^{2}\right)^{n-u} A^{(u)}-\left(-a_{J}^{2}\right)^{n} A^{(0)}\right] \\
& =\prod_{\mu=0}^{n}\left(y_{\mu}^{2}-a_{J}^{2}\right)-\left(-a_{J}^{2}\right)^{n+1}
\end{aligned}
$$


where in the last line we used the definition $y_{0}^{2} \equiv 0$. The contribution of the last expression of $S_{1}$ to $B$ is a constant, $B_{0}$, which is desirable. Therefore, by applying the change

$$
\begin{aligned}
& I=M-1, \quad a_{I}=d_{M}, \\
& \mu=\rho-1, \quad y_{\mu}=z_{\rho}
\end{aligned}
$$

to $B$, we have

$B-B_{0}=\frac{\left(r_{h}^{2}+a_{0}^{2}\right)}{2} \sum_{M=1}^{n+1}\left[\frac{\prod_{\rho=1}^{n+1}\left(z_{\rho}^{2}-d_{M}^{2}\right)}{\left(z_{n+1}^{2}-d_{M}^{2}\right)}\right] \frac{1}{\prod_{N=1}^{n+1}\left(d_{M}^{2}-d_{N}^{2}\right)}$.

Then, using the identity (C5), the expression in the bracket can be expanded in powers of $\left(-d_{M}^{2}\right)$. Using the identity (C1) for the summation over $M$ simplifies $B$ significantly and leads to

$$
B-B_{0}=\frac{\left(r_{h}^{2}+a_{0}^{2}\right)}{2}
$$

which is obviously constant and can be absorbed by $C_{0}$. Therefore, the near-horizon expansion of $b_{\tau}$ starts from $\rho$, as explained in Eq. (35), and gives

$$
\tilde{b}=\tilde{b}_{0} \rho d \tau+\sum_{i=1}^{n} \tilde{b}_{i} d \varphi_{i}
$$

where

$$
\tilde{b}_{0} \equiv \beta r_{h} \sum_{k=0}^{n-1}\left[\frac{\Omega^{i}}{\varepsilon_{i}}\left(-a_{i}^{2}\right)^{n-k}-\frac{(-1)^{n}}{\Xi}\left(-g^{2}\right)^{k}\right] \tilde{A}_{n}^{(k)} .
$$

\section{APPENDIX C: USEFUL IDENTITIES}

$$
\begin{gathered}
\sum_{\alpha=1}^{N} \frac{\left(-x_{\alpha}^{2}\right)^{N-1-q}}{\prod_{\beta=1}^{N}{ }^{\prime}\left(x_{\beta}^{2}-x_{\alpha}^{2}\right)}=\delta_{q, 0}, \\
\sum_{\alpha=1}^{N} \frac{A_{\alpha}^{(p)}}{x_{\alpha}^{2} \prod_{\beta}^{\prime}\left(x_{\beta}^{2}-x_{\alpha}^{2}\right)}=\frac{A^{(p)}}{A^{(N)}}, \\
\frac{1}{\prod_{\alpha=1}^{N}\left(x_{\alpha}^{2}+\lambda\right)}=\sum_{\alpha=1}^{N} \frac{1}{\prod_{\beta}{ }^{\prime}\left(x_{\beta}^{2}-x_{\alpha}^{2}\right)} \frac{1}{\left(x_{\alpha}^{2}+\lambda\right)}, \\
\sum_{\alpha=1}^{N} \frac{A_{\alpha}^{(p)}\left(-x_{\alpha}^{2}\right)^{N-1-q}}{\prod_{\beta}{ }^{\prime}\left(x_{\beta}^{2}-x_{\alpha}^{2}\right)}=\delta_{q, p}, \quad q=1, \ldots N-1, \\
\prod_{\beta=1}^{N}\left(x_{\beta}^{2}+\lambda\right)=\sum_{k=0}^{N} A^{(k)} \lambda^{N-k}, \\
\frac{\prod_{\beta=1}^{N}\left(x_{\beta}^{2}+\lambda\right)}{\left(x_{\alpha}^{2}+\lambda\right)}=\sum_{k=0}^{N-1} A_{\alpha}^{(k)} \lambda^{N-1-k} .
\end{gathered}
$$

[1] B. Carter, Global structure of the Kerr family of gravitational fields, Phys. Rev. 174, 1559 (1968); Hamilton-Jacobi and Schrodinger separable solutions of Einstein's equations, Commun. Math. Phys. 10, 280 (1968); M. Walker and R. Penrose, On quadratic first integrals of the geodesic equations for type [22] spacetimes, Commun. Math. Phys. 18, 265 (1970).

[2] D. Kubiznak, Hidden symmetries of higher-dimensional rotating black holes, arXiv:0809.2452.

[3] V.P. Frolov and D. Kubiznak, Hidden Symmetries of Higher-Dimensional Rotating Black Holes, Phys. Rev. Lett. 98, 011101 (2007); D. Kubiznak and V. P. Frolov, Hidden symmetry of higher dimensional Kerr-NUTAdS spacetimes, Classical Quantum Gravity 24, F1 (2007).

[4] P. Krtous, D. Kubiznak, D. N. Page, and V. P. Frolov, Killing-Yano tensors, Rank-2 Killing tensors, and conserved quantities in higher dimensions, J. High Energy Phys. 02 (2007) 004.
[5] D. Kubiznak and P. Krtous, On conformal Killing-Yano tensors for Plebanski-Demianski family of solutions, Phys. Rev. D 76, 084036 (2007).

[6] M. Cariglia, P. Krtous, and D. Kubiznak, Dirac equation in Kerr-NUT-(A)dS spacetimes: Intrinsic characterization of separability in all dimensions, Phys. Rev. D 84, 024008 (2011).

[7] V. P. Frolov, P. Krtous, and D. Kubiznak, Separability of Hamilton-Jacobi and Klein-Gordon equations in General Kerr-NUT-AdS spacetimes, J. High Energy Phys. 02 (2007) 005; P. Krtous, D. Kubiznak, D. N. Page, and M. Vasudevan, Constants of geodesic motion in higherdimensional black-hole spacetimes, Phys. Rev. D 76, 084034 (2007); Complete Integrability of Geodesic Motion in General Kerr-NUT-AdS Spacetimes, Phys. Rev. Lett. 98, 061102 (2007).

[8] O. Lunin, Maxwell's equations in the Myers-Perry geometry, J. High Energy Phys. 12 (2017) 138; P. Krtouš, V. P. Frolov, and D. Kubiznak, Separation of Maxwell equations 
in Kerr-NUT-(A)dS spacetimes, Nucl. Phys. B934, 7 (2018).

[9] V. Frolov, P. Krtous, and D. Kubiznak, Black holes, hidden symmetries, and complete integrability, Living Rev. Relativity 20, 6 (2017).

[10] D. Astefanesei, K. Goldstein, R. P. Jena, A. Sen, and S. P. Trivedi, Rotating attractors, J. High Energy Phys. 10 (2006) 058 .

[11] D. Astefanesei and H. Yavartanoo, Stationary black holes and attractor mechanism, Nucl. Phys. B794, 13 (2008).

[12] J. M. Bardeen and G. T. Horowitz, The extreme Kerr throat geometry: A vacuum analog of $\mathrm{AdS}_{2} \times S^{2}$, Phys. Rev. D 60 , 104030 (1999); H. K. Kunduri, J. Lucietti, and H. S. Reall, Near-horizon symmetries of extremal black holes, Classical Quantum Gravity 24, 4169 (2007); H. K. Kunduri and J. Lucietti, A classification of near-horizon geometries of extremal vacuum black holes, J. Math. Phys. (N.Y.) 50, 082502 (2009); H. K. Kunduri and J. Lucietti, Classification of near-horizon geometries of extremal black holes, Living Rev. Relativity 16, 8 (2013).

[13] P. Figueras, H. K. Kunduri, J. Lucietti, and M. Rangamani, Extremal vacuum black holes in higher dimensions, Phys. Rev. D 78, 044042 (2008).

[14] P. Claus, M. Derix, R. Kallosh, J. Kumar, P. K. Townsend, and A. Van Proeyen, Black Holes and Superconformal Mechanics, Phys. Rev. Lett. 81, 4553 (1998).

[15] A. Galajinsky, Particle dynamics on $\mathrm{AdS}_{2} \times S^{2}$ background with two-form flux, Phys. Rev. D 78, 044014 (2008); Particle dynamics near extreme Kerr throat and supersymmetry, J. High Energy Phys. 11 (2010) 126; A. Galajinsky and A. Nersessian, Conformal mechanics inspired by extremal black holes in $d=4$, J. High Energy Phys. 11 (2011) 135; A. Galajinsky and K. Orekhov, $N=2$ superparticle near horizon of extreme Kerr-Newman-AdS-dS black hole, Nucl. Phys. B850, 339 (2011); S. Bellucci, A. Nersessian, and V. Yeghikyan, Action-angle variables for the particle near extreme Kerr throat, Mod. Phys. Lett. A 27, 1250191 (2012); A. Galajinsky, A. Nersessian, and A. Saghatelian, Superintegrable models related to near horizon extremal Myers-Perry black hole in arbitrary dimension, J. High Energy Phys. 06 (2013) 002.

[16] M. M. Sheikh-Jabbari and H. Yavartanoo, EVH black holes, $\mathrm{AdS}_{3}$ throats and EVH/CFT proposal, J. High Energy Phys. 10 (2011) 013.

[17] J. de Boer, M. Johnstone, M. M. Sheikh-Jabbari, and J. Simon, Emergent IR dual 2D CFTs in charged $\mathrm{AdS}_{5}$ black holes, Phys. Rev. D 85, 084039 (2012); H. Golchin, M. M. Sheikh-Jabbari, and A. Ghodsi, Dual 2D CFT identification of extremal black rings from holes, J. High Energy Phys. 10 (2013) 194.

[18] S. Sadeghian and M. H. Vahidinia, $\mathrm{AdS}_{3}$ to $\mathrm{dS}_{3}$ transition in the near horizon of asymptotically de Sitter solutions, Phys. Rev. D 96, 044004 (2017).

[19] S. Sadeghian, M. M. Sheikh-Jabbari, M. H. Vahidinia, and H. Yavartanoo, Three theorems on near horizon extremal vanishing horizon geometries, Phys. Lett. B 753, 488 (2016); Near horizon structure of extremal vanishing horizon black holes, Nucl. Phys. B900, 222 (2015).

[20] Y. Mitsuka and G. Moutsopoulos, No more CKY two-forms in the NHEK, Classical Quantum Gravity 29, 045004 (2012).

[21] J. Rasmussen, On hidden symmetries of extremal KerrNUT-AdS-dS black holes, J. Geom. Phys. 61, 922 (2011).

[22] J. $\mathrm{Xu}$ and R. H. Yue, On hidden symmetries of $d>4$ NHEK-N-AdS geometry, Commun. Theor. Phys. 63, 31 (2015); D. Chernyavsky, Reducibility of Killing tensors in $d>4$ NHEK geometry, J. Geom. Phys. 83, 12 (2014).

[23] I. Kolar and P. Krtous, NUT-like and near-horizon limits of Kerr-NUT-(A)dS spacetimes, Phys. Rev. D 95, 124044 (2017).

[24] T. Hakobyan, A. Nersessian, and M. M. Sheikh-Jabbari, Near horizon extremal Myers-Perry black holes and integrability of associated conformal mechanics, Phys. Lett. B 772, 586 (2017).

[25] H. Demirchian, Note on constants of motion in conformal mechanics associated with near horizon extremal MyersPerry black holes, Mod. Phys. Lett. A 32, 1750144 (2017).

[26] H. Demirchian, A. Nersessian, S. Sadeghian, and M. M. Sheikh-Jabbari, Integrability of geodesics in near-horizon extremal geometries: Case of Myers-Perry black holes in arbitrary dimensions, Phys. Rev. D 97, 104004 (2018); in Proceedings of the Conference SYMPHYS-XVII (to be published).

[27] W. Chen, H. Lu, and C. N. Pope, General Kerr-NUT-AdS metrics in all dimensions, Classical Quantum Gravity 23, 5323 (2006).

[28] S. Mukherjee, S. Chakraborty, and N. Dadhich, On some novel features of the Kerr-Newman-NUT spacetime, arXiv:1807.02216.

[29] R. C. Myers and M. J. Perry, Black holes in higher dimensional space-times, Ann. Phys. (N.Y.) 172, 304 (1986); R. C. Myers, Myers-Perry black holes, arXiv:1111.1903. 